\title{
Diversity Rate of Change Measurement for Particle Swarm Optimisers
}

\author{
Phlippie Bosman and Andries Engelbrecht \\ Department of Computer Science, University of Pretoria, Pretoria, South Africa \\ pbosman@cs.up.ac.za, engel@cs.up.ac.za
}

\begin{abstract}
The diversity of a particle swarm can reflect the swarm's explorative/exploitative behaviour at a given time step. This paper proposes a diversity rate of change measure to quantify the rate at which particle swarms decrease their diversity over time. The proposed measure is based on a two-piecewise linear approximation of diversity measurements sampled at regular time steps. The proposed measure is the slope of the first of the two lines. It is shown that, when comparing the measure among different algorithms, the measure reflects the differences in the behaviour of algorithms in terms of their exploration-exploitation trade-off. The measure can potentially be used to characterise and classify different algorithms based on algorithm behaviour.
\end{abstract}

\section{Introduction}

Particle swarm optimisation (PSO) is a stochastic optimisation algorithm that maintains a swarm of particles, where each particle represents a candidate solution. An important characteristic that describes the search behaviour of a PSO algorithm (and other population-based algorithms) is diversity. The diversity of a swarm is the degree of dispersion of the swarm's particles [15].

Diversity is related to the notions of exploration and exploitation: the more diverse a swarm is, the more its particles are dispersed over the search space, and the more the swarm is exploring. Measuring diversity, then, can give an indication of an algorithm's search behaviour at a certain time step. Considering diversity measures over time can give an indication of the rate at which a swarm converges, or alternatively, the rate at which a swarm moves from an explorative to an exploitative behaviour, which has an impact on the performance of the algorithm.

A single, measurable value that reflects an algorithm's behaviour with regards to the rate at which diversity decreases over time can potentially be used to classify algorithms in different behavioural classes based on the rate at which the algorithms move from exploration to exploitation. Such a measure of diversity rate-of-change can potentially be used to predict performance for the different behavioural algorithm classes. This paper proposes a measure that can be used to differentiate different algorithms in terms of their behaviour with regards to the rate at which diversity decreases. To the knowledge of the authors, this is the first 
such measure. The proposed diversity rate-of-change (DRoC) measure is based on a two-piecewise linear approximation of the instantaneous diversity measures, computed at regular time steps: the slope of the first line of the piecewise linear approximation is used as the DRoC measure. A lower negative value for the slope indicates that an algorithm's diversity decreases faster, that the algorithm spends less time exploring, and that its particles converge to a smaller region faster.

The DRoC values for a number of PSO algorithms are computed in this paper for a large set of benchmark functions. These values are then used in the empirical section to see if the DRoC values can be used to characterise the rate at which search behaviour changes from exploration to exploitation, and to see if groups of algorithms can be found that exhibit the same behaviour.

The rest of the paper is organised as follows: Section 2 provides background on PSO, the algorithms used, diversity measures, and linear approximations. Section 3 lists expectations with reference to the rate at which diversity should decrease for the different algorithms. Section 4 presents the proposed measure. Section 5 summarises the experimental procedure. Section 6 provides and discusses the results.

\section{Background}

This section provides background information on the main concepts used.

\subsection{Particle Swarm Optimisers}

The basic PSO algorithm, introduced by Kennedy and Eberhart in 1995 [5, 10], is a population-based search algorithm inspired from the behaviour of birds in flocks. A PSO algorithm maintains a swarm of particles, where each particle represents a candidate solution to an optimisation problem.

The original (gbest) PSO [10] updates the position of each particle $\mathbf{x}_{i}$ by adding a velocity, or step size, $\mathbf{v}_{i}$ to the particle's previous position as follows:

$$
\mathbf{x}_{i}(t+1)=\mathbf{x}_{i}(t)+\mathbf{v}_{i}(t+1) .
$$

The velocity update for each particle consists of three components: the momentum component, which is a fraction of the particle's velocity at the previous time step; the cognitive component, which pulls the particle to a so-called personal best; and a social component, which pulls the particle towards a global best. The velocity update is as follows:

$$
\mathbf{v}_{i}(t+1)=\omega \mathbf{v}_{i}(t)+c_{1} \mathbf{r}_{1}(t)\left[\overline{\mathbf{y}}_{i}(t)-\mathbf{x}_{i}(t)\right]+c_{2} \mathbf{r}_{2}(t)\left[\hat{\mathbf{y}}(t)-\mathbf{x}_{i}(t)\right],
$$

where $\omega$ is the inertia weight, $c_{1}$ and $c_{2}$ are constants, and $\mathbf{r}_{1}$ and $\mathbf{r}_{2}$ are vectors of random numbers sampled from the uniform distribution $U(0,1)$.

The cognitive component is a weighted difference between $\bar{y}_{i}(t)$, the personal best position visited by particle $i$ up to time step $t$, and its current position. 
The effect of the component in the update equation is that the particle is drawn towards its personal best position. Similarly, the social component draws the particle in the direction of $\hat{\mathbf{y}}(t)$, the global best position found by the swarm.

\subsection{Particle Swarm Optimiser Variations}

Many variations of the basic PSO exist. Variations that are used in this study are described in this section.

Some variation arises from introducing a notion of neighbourhoods. The original PSO velocity update (Equation 2) can be changed to

$$
\mathbf{v}_{i}(t+1)=\omega \mathbf{v}_{i}(t)+c_{1} \mathbf{r}_{1}(t)\left[\overline{\mathbf{y}}_{i}(t)-\mathbf{x}_{i}(t)\right]+c_{2} \mathbf{r}_{2}(t)\left[\hat{\mathbf{y}}_{i}(t)-\mathbf{x}_{i}(t)\right],
$$

where $\hat{\mathbf{y}}_{i}(t)$ is particle $i$ 's local best position, which is the best position found in that particle's neighbourhood. A neighbourhood is a topology which connects each particle to some other particles; different topologies result in different variations of the PSO. Neighbourhoods have an inhibiting effect on information flow, since particles can, at each iteration of the algorithm, only gain information about local best positions that is already available to their direct neighbours.

The basic gbest PSO uses a star topology where each particle is connected to every other particle. Information flow is not inhibited, and information flow is instant.

The local best (lbest) PSO is a common variation of the gbest PSO that uses a ring topology instead of a star topology $[5,19]$, such that each particle is only connected to two other particles: its index-wise predecessor and successor. The longest path between two particles is half the size of the swarm, so at most $n_{s} / 2$ iterations might be required for information to pass from one particle to another. Information flow is therefore quite slow.

The Von Neumann topology [11] is an intermediate topology where particles are usually logically arranged on a 2-D grid. Information flow in Von Neumann PSO is slower than in gbest PSO but faster than in lbest PSO.

The basic PSO has a potential problem: if $\mathbf{x}_{i}=\overline{\mathbf{y}}_{i}=\hat{\mathbf{y}}_{i}$ for a particle, that particle's update depends only on its previous velocity. This can cause the algorithm to stagnate on the swarm's global best position, even if that position is not a local optimum [6]. The guaranteed convergence PSO (GCPSO) [1] overcomes this problem by using an altered position and velocity update equation for the global best particle, which forces that particle to search for a better position in a confined region around the global best position.

The GCPSO can be used with neighbourhood topologies such as star, ring and Von Neumann. Neighbourhoods have a similar effect in the GCPSO [16] as they do in the standard PSO.

Particles converge to a weighted average between their personal and local best positions [2], referred to in this paper as the theoretical attractor point. Kennedy [9] has proposed that the entire velocity update equation be replaced by a random number sampled from a Gaussian distribution around the theoretical attractor point, with a deviation the magnitude of the distance between the 
personal and global best. The resultant algorithm is called the barebones PSO (BBPSO). Kennedy also proposed [9] an alternative barebones PSO (aBBPSO), where the particle sampled from the above Gaussian distribution is recombined with the particle's personal best position.

The social PSO (SPSO) is a variation of the gbest PSO where the velocity update does not contain a cognitive component. The particles are only guided by the global best position and their own previous velocity. The particles converge towards the global best position, rather than a weighted average between that and their personal best positions, leading to very fast convergence.

\subsection{Swarm Diversity}

The diversity of a swarm is the degree of dispersion of its particles. Many existing diversity measures were investigated by Olorunda and Engelbrecht [15]. Note that these measures are instantaneous and thus only measure a swarm's diversity at a single time step. The two measures found in [15] to be the most accurate are the average distance around the swarm centre, and the average distance around all particles in the swarm. The average distance around the swarm centre was used in this study, given by

$$
\mathcal{D}=\frac{1}{n_{s}} \sum_{i=1}^{n_{s}} \sqrt{\sum_{k=1}^{n_{x}}\left(x_{i k}-\bar{x}_{k}\right)^{2}},
$$

where $n_{s}$ is the swarm size, $n_{x}$ is the number of dimensions of the problem, $x_{i k}$ is the $k$-th dimension of the $i$-th particle position, and $\bar{x}_{k}$ is the average of the $k$-th dimension over all particles.

\subsection{Two-Piecewise Linear Approximation}

A two-piecewise linear approximation of a function,

$$
y(x) \approx f(x) \text { for } i_{0} \leq x \leq i_{2},
$$

is a mapping of two line segments, taking the form

$$
y(x)= \begin{cases}a_{1}+b_{1} x & \text { for } i_{0} \leq x \leq i_{1} \\ a_{2}+b_{2} x & \text { for } i_{1}<x \leq i_{2}\end{cases}
$$

where $a_{j}$ and $b_{j}$ are the $y$ intersection and the gradient of the $j$-th line segment, respectively. The mapping is a minimisation problem aimed at finding optimal values for $a_{1}, a_{2}, b_{1}, b_{2}$, and $i_{1}$. The goal of the mapping is to minimise the least squares error (LSE) between the function and the linear approximation, given by

$$
L S E=\sum_{x=i_{0}}^{i_{2}}(f(x)-y(x))^{2}
$$




\section{Algorithm behaviour}

Engelbrecht [7] found that different algorithms exhibit different diversity profiles, with reference to the rate at which diversity is decreased. Preliminary expectations about the rate at which different algorithms reduce diversity, and how this behaviour differs among different algorithms, can be made based on observations published in PSO literature and based on the definitions of position and velocity updates. This section discusses these expectations.

Because neighbourhoods directly influence information flow, it is expected that more connected neighbourhoods will converge more quickly [11,12], and so that their diversity will decrease more quickly. Therefore, when comparing any PSO algorithm that uses a star topology with the same algorithm using a ring or Von Neumann topology, it is expected that the rate of decrease in diversity for the PSO that uses the star topology will be faster than for the other algorithms. It is also expected that algorithms that use a Von Neumann topology will reduce diversity faster than algorithms that use a ring topology.

When comparing the SPSO with any of the other algorithms discussed in Section 2.2 , it is expected that the SPSO will reduce diversity at a much faster rate due to the lack of the cognitive component, which is the component that facilitates exploration [7].

Due to the local search around the neighbourhood best that the GCPSO does, it is expected that the GCPSO reduces diversity at a somewhat faster rate than a basic PSO with a corresponding neighbourhood topology, but not at a significantly different rate.

When comparing the BBPSO with the aBBPSO, it is expected that the aBBPSO reduces diversity at a slower rate due to the random combination of the personal best position in the position update. This is expected to strengthen the cognitive-guided behaviour of particles, and weaken their social behaviour, delaying the swarm's convergence towards the theoretical attractor point.

\section{Diversity Rate-of-Change Measure}

Analysis of diversity measurements taken at regular time steps revealed a common pattern in the diversity profiles of the PSO algorithms studied in this paper, as illustrated in Figure 1: The initial diversity value is very high, due to particles being randomly initialised over the search space. The diversity shows a trend of rapid decrease for a number of iterations, referred to as phase one in this paper. This decrease is due to particles converging on a promising region of the search space. After the first phase, the diversity still generally decreases, though at a slower rate than in phase one, representing exploitation of the promising region in order to locate a good solution. This is referred to as the second phase. The pattern reflects a common behaviour in many PSO algorithms: that exploration is initially high, but then gives way to exploitation. (Of the PSO algorithms included in this study, all find a single solution in a static environment, and none have processes implemented through which the diversity of their swarms are managed.) 


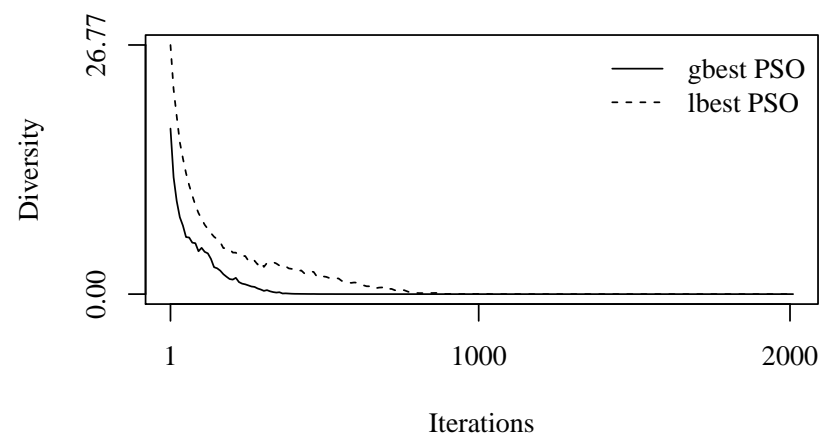

Fig. 1. Average diversity measurements over time for the gbest (solid line) and lbest (dashed line) PSO on Levy's function

A two-piecewise linear approximation of diversity measurements produces one line with a slope that is relatively larger than the second line. An example of such a two-piecewise linear approximation is shown in Figure 2. The slope of the first line, representing phase one, quantifies the rate at which diversity decreases, i.e. the rate at which the swarm moves from an explorative to exploitative behaviour. The DRoC measure proposed in this paper is therefore simply the slope of this first line.

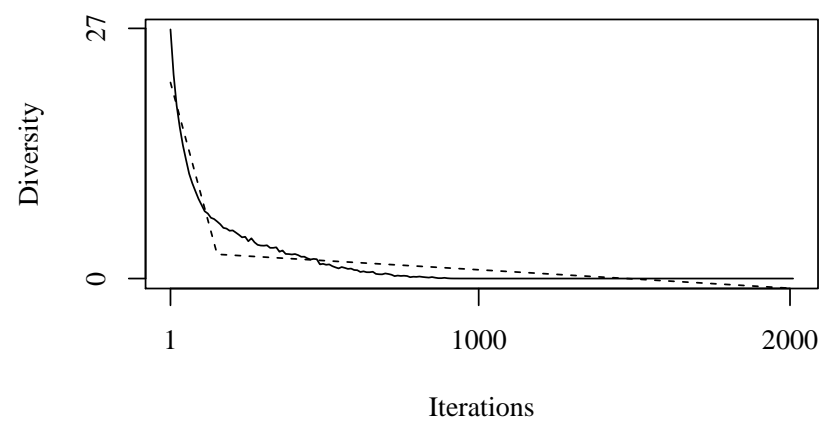

Fig. 2. Average diversity measurements over time for the gbest PSO over Levy's function (solid line) with two-piecewise linear approximation (dashed line).

Because the DRoC measure relies on diversity measurements over the entire run of a simulation, the proposed DRoC measure must be calculated after a simulation has completed. 


\section{Experimental Procedure}

This section describes the experimental procedure followed to evaluate the DRoC measure.

Different PSO algorithms were run on a selection of benchmark functions using CILib, ${ }^{1}$ an open-source framework for testing computational intelligence algorithms. The algorithms used, and the corresponding control parameters, are listed in Table 1. The benchmark functions are summarised in table 2. Each algorithm was used with 25 particles. For each function, each algorithm was run 30 times from different random initial conditions, for 2000 iterations. Diversity measures were sampled at every 10 iterations.

Table 1. Algorithms and control parameters used in this study

\begin{tabular}{|l|l|}
\hline Algorithm name & Control parameters \\
\hline \hline Lbest PSO & Ring neighbourhood topology \\
\hline Gbest PSO & Star neighbourhood topology \\
\hline Von Neumann PSO (V.N. PSO) & Von Neumann neighbourhood topology \\
\hline Lbest GCPSO* & Ring neighbourhood topology \\
\hline Gbest GCPSO & Star neighbourhood topology \\
\hline $\begin{array}{l}\text { Von Neumann GCPSO } \\
(\text { V.N. GCPSO) }\end{array}$ & Von Neumann neighbourhood topology \\
\hline BBPSO & \\
\hline aBBPSO & Star neighbourhood topology \\
\hline SPSO & $\begin{array}{l}\text { Star neighbourhood topology } \\
\text { Probability of combination }=0.5\end{array}$ \\
\hline$* \omega=0.729844, c_{1}=1.49618, c_{2}=1.49618$ \\
\hline
\end{tabular}

For each benchmark function, a pair-wise Mann-Whitney U test with a $95 \%$ level of significance was performed on each pair of algorithms in order to determine if significant differences occur in the left slope among the different algorithms. The results were summarised to indicate whether the left slope for the first algorithm of the pair is significantly smaller (-1) or larger (1) than the left slope for the second algorithm, or whether no significant difference exists between the left slopes of the two algorithms (0). It is hypothesised that, where algorithms are intuitively expected to behave differently in terms of the rate of decrease in diversity, this difference will be reflected by the results of the U tests.

\section{Results}

The results from all pair-wise $\mathrm{U}$ tests are summarised in Table 6 . The summarised Mann-Whitney $U$ test result is shown for each algorithm pair (columns) and each

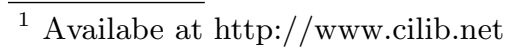


Table 2. Benchmark functions used in this study

\begin{tabular}{|l|r|r|}
\hline Function name & Domain & Dimensions \\
\hline \hline Ackley [21] & $x_{i} \epsilon[-32,32]$ & 25 \\
\hline Alpine [18] & $x_{i} \epsilon[-10,10]$ & 25 \\
\hline Eggholder[13] & $x_{i} \epsilon[-512,512]$ & 25 \\
\hline Elliptic [20] & $x_{i} \epsilon[-100,100]$ & 25 \\
\hline Goldstein-Price [21] & $x_{i} \epsilon[-2,2]$ & 2 \\
\hline Griewank [21] & $x_{i} \epsilon[-600,600]$ & 25 \\
\hline Levy [13] & $x_{i} \epsilon[-10,10]$ & 25 \\
\hline Michalewicz [3] & $x_{i} \epsilon[0, \pi]$ & 25 \\
\hline Quadric [21] & $x_{i} \epsilon[-100,100]$ & 25 \\
\hline Quartic [21] & $x_{i} \epsilon[-1.28,1.28]$ & 25 \\
\hline Rastrigin [21] & $x_{i} \epsilon[-5.12,5.12]$ & 25 \\
\hline Rosenbrock [21] & $x_{i} \epsilon[-2.048,2.048]$ & 25 \\
\hline Salomon [17] & $x_{i} \epsilon[-100,100]$ & 25 \\
\hline Schwefel 1.2 & $x_{i} \epsilon[-100,100]$ & 25 \\
\hline Schwefel 2.22 [21] & $x_{i} \epsilon[-10,10]$ & 25 \\
\hline Schwefel 2.26 [21] & $x_{i} \epsilon[-500,500]$ & 25 \\
\hline Six-hump camel-back $[21]$ & $x_{i} \epsilon[-5,5]$ & 2 \\
\hline Spherical [4] & $x_{i} \epsilon[-100,100]$ & 25 \\
\hline Step [21] & $x_{i} \epsilon[-20,20]$ & 25 \\
\hline Zakharov [8] & $x_{i} \epsilon[-5,10]$ & 25 \\
\hline
\end{tabular}

function (rows). The final 3 rows respectively provide the number of each result for each algorithm pair.

As expected, the gbest PSO's measures indicate that it usually converged at a faster rate than the lbest PSO. Similarly, the GCPSO with a star topology usually converged faster than the GCPSO with a ring topology. In both the standard PSO and the GCPSO, the Von Neumann variations often produced no significant difference from the gbest variations, but the Von Neumann variations usually converged faster than the lbest variations.

When comparing the standard PSO algorithms with the GCPSO algorithms using the same neighbourhood topologies, no difference was found for most of the functions, as expected. For the few differences found, the GCPSO algorithms decreased diversity at a slower rate than the corresponding standard PSO algorithms, contrary to expectations. This may be an indication that the fitness landscape plays a role in the DRoC; this possibility is also observed by Engelbrecht in [7].

The SPSO usually decreased its diversity at a faster rate than any other algorithm, as expected.

Comparison of the BBPSO with the aBBPSO supports the expectation that the variation to BBPSO should converge more slowly, though there was often no significant difference between the two.

Both the gbest PSO and the gbest GCPSO were usually not significantly different when compared to the BBPSO. For most of the significant differences 
Table 3. Summarised results of pair-wise Mann-Whitney U tests for each pair of algorithms, for each benchmark function

\begin{tabular}{|c|c|c|c|c|c|c|c|c|c|c|c|c|c|c|c|c|c|c|}
\hline & 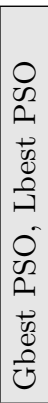 & 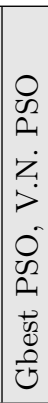 & 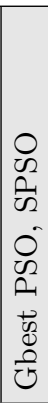 & 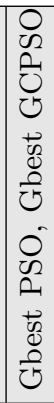 & 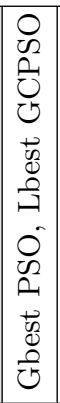 & 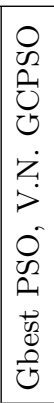 & 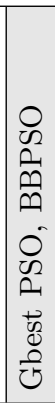 & 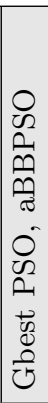 & 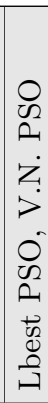 & 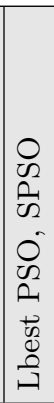 & 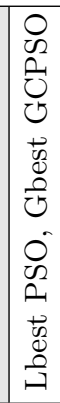 & 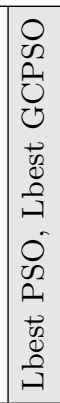 & 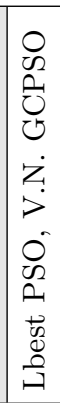 & 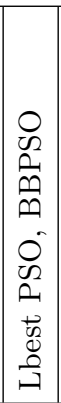 & 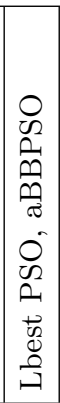 & 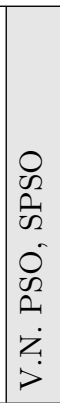 & $\begin{array}{c}0 \\
0 \\
0 \\
0 \\
0 \\
0 \\
0 \\
0 \\
0 \\
0 \\
0 \\
0 \\
\tilde{D} \\
2 \\
z \\
1 \\
>\end{array}$ & 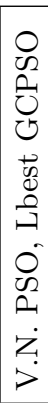 \\
\hline 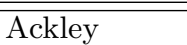 & -1 & $\overline{0}$ & 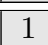 & $\overline{0}$ & -1 & 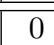 & $\overline{0}$ & $\overline{11}$ & 1 & 1 & 1 & 0 & 1 & 1 & 1 & 1 & 0 & $\mid-1$ \\
\hline Alpine & -1 & -1 & 1 & 0 & $\mid-1$ & -1 & 0 & -1 & 0 & 1 & 1 & 0 & 1 & 1 & 0 & 1 & 1 & 0 \\
\hline Ellip & 1 & 1 & 1 & 0 & 0 & 1 & 1 & 1 & 1 & 1 & -1 & -1 & 1 & 1 & 1 & 1 & -1 & -1 \\
\hline Eggh & -1 & -1 & 1 & 0 & -1 & \begin{tabular}{|l|}
-1 \\
\end{tabular} & 0 & -1 & 1 & 1 & 1 & 0 & 1 & 1 & 0 & 1 & 1 & -1 \\
\hline $\begin{array}{l}\text { Goldstein- } \\
\text { Price }\end{array}$ & 1 & 1 & 1 & 0 & 1 & 1 & 1 & 0 & 0 & 0 & -1 & 0 & 0 & 0 & -1 & 0 & -1 & \begin{tabular}{|l|}
-1 \\
\end{tabular} \\
\hline Griewank & -1 & 0 & 1 & 0 & -1 & 0 & 0 & 1 & 1 & 1 & 1 & 0 & 1 & 1 & 1 & 1 & 0 & -1 \\
\hline Levy & 1 & 0 & 1 & 0 & 1 & 0 & 1 & 1 & 0 & 1 & -1 & 0 & 0 & 1 & 1 & 1 & -1 & 0 \\
\hline Michalewic & -1 & -1 & 1 & -1 & -1 & \begin{tabular}{|l|}
-1 \\
\end{tabular} & 0 & -1 & 1 & 1 & 1 & 0 & 1 & 1 & 1 & 1 & 1 & -1 \\
\hline Quadric & \begin{tabular}{|l|}
-1 \\
\end{tabular} & -1 & 1 & 0 & \begin{tabular}{|l|}
-1 \\
\end{tabular} & \begin{tabular}{|l|}
-1 \\
\end{tabular} & 0 & -1 & 1 & 1 & 1 & 0 & 1 & 1 & 0 & 1 & 1 & \begin{tabular}{|l|}
-1 \\
\end{tabular} \\
\hline Qual & -1 & 0 & 1 & 0 & $\mid-1$ & 0 & 0 & 1 & 1 & 1 & 0 & 0 & 0 & 1 & 1 & 1 & -1 & \begin{tabular}{|l|}
-1 \\
\end{tabular} \\
\hline Ras & -1 & -1 & 1 & 0 & -1 & -1 & 0 & -1 & 0 & 1 & 1 & 0 & 0 & 1 & -1 & 1 & 1 & 0 \\
\hline Rosenbrock & -1 & 0 & 1 & -1 & -1 & \begin{tabular}{|l|}
-1 \\
\end{tabular} & 0 & 0 & 1 & 1 & 1 & 0 & 1 & 1 & 1 & 1 & -1 & -1 \\
\hline Salom & -1 & 0 & 1 & 0 & \begin{tabular}{|l|}
-1 \\
\end{tabular} & 0 & 0 & 0 & 1 & 1 & 0 & 0 & 1 & 0 & 0 & 1 & 0 & -1 \\
\hline Schwefel 1.2 & -1 & -1 & 1 & $\overline{0}$ & \begin{tabular}{|l|}
-1 \\
\end{tabular} & \begin{tabular}{|l|}
-1 \\
\end{tabular} & 0 & -1 & 1 & 1 & 1 & 0 & 1 & 1 & 0 & 1 & 1 & -1 \\
\hline $\begin{array}{l}\text { Schwefel } \\
2.22\end{array}$ & -1 & -1 & 0 & -1 & -1 & \begin{tabular}{|l|}
-1 \\
\end{tabular} & 1 & 1 & 1 & 1 & 1 & 0 & 1 & 1 & 1 & 1 & 0 & -1 \\
\hline $\begin{array}{l}\text { Schwefel } \\
2.26\end{array}$ & \begin{tabular}{|l|}
-1 \\
\end{tabular} & -1 & 1 & 0 & \begin{tabular}{|l|}
-1 \\
\end{tabular} & \begin{tabular}{|l|}
-1 \\
\end{tabular} & 0 & -1 & 1 & 1 & 1 & 0 & 1 & 1 & 1 & 1 & 1 & \begin{tabular}{|l|}
-1 \\
\end{tabular} \\
\hline Sixhump & 0 & 0 & 1 & 0 & 0 & \begin{tabular}{|l|}
-1 \\
\end{tabular} & 0 & 1 & 0 & 1 & 0 & -1 & -1 & 0 & 1 & 1 & 0 & 0 \\
\hline Spherical & \begin{tabular}{|l}
-1 \\
\end{tabular} & 0 & 1 & 0 & \begin{tabular}{|l|}
-1 \\
\end{tabular} & 0 & 0 & 1 & 1 & 1 & 1 & 0 & 1 & 1 & 1 & 1 & 0 & \begin{tabular}{|l}
-1 \\
\end{tabular} \\
\hline Step & \begin{tabular}{|l}
-1 \\
\end{tabular} & 0 & 1 & 1 & -1 & 0 & 1 & 1 & 1 & 1 & 1 & 0 & 1 & 1 & 1 & 1 & 1 & \begin{tabular}{|l}
-1 \\
\end{tabular} \\
\hline Zakharov & -1 & 0 & 1 & 0 & \begin{tabular}{|l|}
-1 \\
\end{tabular} & 0 & -1 & -1 & 1 & 1 & 1 & 0 & 1 & 0 & -1 & 1 & 0 & \begin{tabular}{|l|}
-1 \\
\end{tabular} \\
\hline Total -1's & 16 & 8 & 0 & 3 & 16 & 10 & 1 & 8 & 0 & 0 & 3 & 2 & 1 & 0 & 3 & 0 & 5 & 16 \\
\hline Total 0's & 1 & 10 & 1 & 16 & 2 & 8 & 14 & 3 & 5 & 1 & 3 & 18 & 4 & 4 & 5 & 1 & 7 & 4 \\
\hline Total 1's & \begin{tabular}{|l}
3 \\
\end{tabular} & 2 & 19 & 1 & 2 & 2 & 5 & 9 & 15 & 19 & 14 & 0 & 15 & 16 & 12 & 19 & 8 & 0 \\
\hline
\end{tabular}

found in both comparisons, the BBPSO decreased its diversity faster. However, comparison of the gbest PSO and the gbest GCPSO to the aBBPSO produced varying results: there was usually a significant difference, but the aBBPSO was found to decrease its diversity faster than the gbest PSO and the gbest GCPSO as often as the aBBPSO did so at a slower rate than the gbest PSO and gbest 


\begin{tabular}{|c|c|c|c|c|c|c|c|c|c|c|c|c|c|c|c|c|c|c|}
\hline & 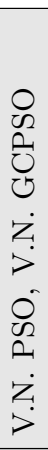 & 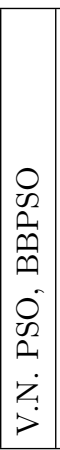 & 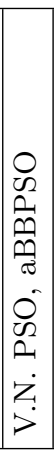 & $\begin{array}{c}0 \\
w \\
0 \\
0 \\
0 \\
\overrightarrow{0} \\
0 \\
0 \\
0 \\
0 \\
0 \\
0 \\
\tilde{w} \\
\tilde{w}\end{array}$ & 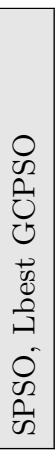 & 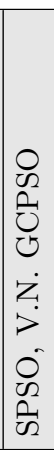 & 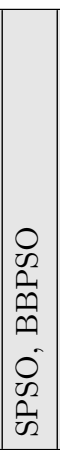 & 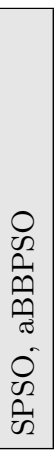 & 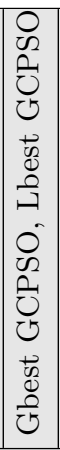 & 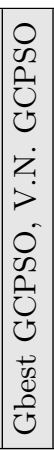 & 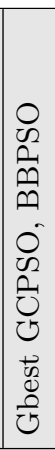 & 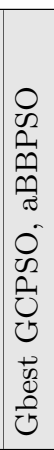 & 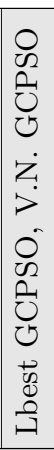 & 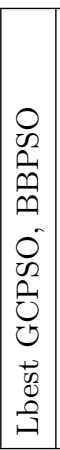 & 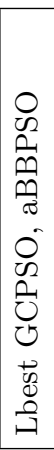 & 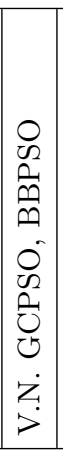 & 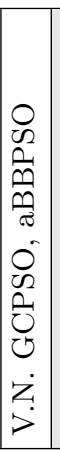 & 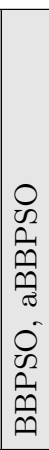 \\
\hline Ackley & 0 & 1 & 1 & \begin{tabular}{|l|}
-1 \\
\end{tabular} & \begin{tabular}{|l|}
-1 \\
\end{tabular} & $\mid-1$ & \begin{tabular}{|l|}
-1 \\
\end{tabular} & -1 & -1 & 0 & 0 & 1 & 1 & 1 & 1 & 1 & 1 & 0 \\
\hline Alpine & 0 & 1 & -1 & \begin{tabular}{|l|}
-1 \\
\end{tabular} & \begin{tabular}{|l|}
-1 \\
\end{tabular} & -1 & -1 & -1 & -1 & -1 & 0 & -1 & 1 & 1 & 0 & 1 & \begin{tabular}{|l|}
-1 \\
\end{tabular} & -1 \\
\hline Elliptic & 0 & 1 & 1 & -1 & -1 & -1 & -1 & -1 & 1 & 1 & 1 & 1 & 1 & 1 & 1 & 1 & 1 & 0 \\
\hline Eggholder & 0 & 1 & \begin{tabular}{|l|}
-1 \\
\end{tabular} & \begin{tabular}{|l|}
-1 \\
\end{tabular} & -1 & -1 & \begin{tabular}{|l|}
-1 \\
\end{tabular} & -1 & -1 & -1 & 0 & -1 & 1 & 1 & 0 & 1 & \begin{tabular}{|l|}
-1 \\
\end{tabular} & -1 \\
\hline $\begin{array}{l}\text { Goldstein- } \\
\text { Price }\end{array}$ & -1 & 0 & -1 & \begin{tabular}{|l|}
-1 \\
\end{tabular} & 0 & -1 & 0 & -1 & 1 & 1 & 1 & 0 & 0 & 0 & \begin{tabular}{|l|}
-1 \\
\end{tabular} & 0 & \begin{tabular}{|l|}
-1 \\
\end{tabular} & -1 \\
\hline Griewank & 0 & 1 & 1 & 0 & \begin{tabular}{|l|}
-1 \\
\end{tabular} & -1 & 0 & 0 & -1 & 0 & 0 & 0 & 1 & 1 & 1 & 0 & 1 & 0 \\
\hline Levy & 0 & 1 & 1 & -1 & -1 & -1 & 0 & -1 & 1 & 1 & 1 & 1 & 0 & 1 & 1 & 1 & 1 & 0 \\
\hline Michalewicz & 0 & 1 & 0 & -1 & -1 & -1 & -1 & -1 & -1 & -1 & 1 & -1 & 1 & 1 & 1 & 1 & 0 & -1 \\
\hline Quadric & 0 & 1 & -1 & \begin{tabular}{|l|}
-1 \\
\end{tabular} & -1 & -1 & -1 & -1 & -1 & -1 & 0 & -1 & 1 & 1 & 0 & 1 & -1 & -1 \\
\hline Quartic & -1 & 0 & 1 & -1 & -1 & -1 & -1 & -1 & -1 & 0 & 1 & 1 & 1 & 1 & 1 & 1 & 1 & 1 \\
\hline Rastrigin & 0 & 1 & -1 & \begin{tabular}{|l|}
-1 \\
\end{tabular} & -1 & -1 & -1 & -1 & -1 & -1 & 0 & -1 & 0 & 1 & \begin{tabular}{|l|}
-1 \\
\end{tabular} & 1 & \begin{tabular}{|l|}
-1 \\
\end{tabular} & -1 \\
\hline Rosenbrock & 0 & 0 & 0 & \begin{tabular}{|l|}
-1 \\
\end{tabular} & -1 & -1 & -1 & -1 & -1 & 0 & 1 & 0 & 1 & 1 & 1 & 1 & 0 & -1 \\
\hline Salomon & 0 & 0 & -1 & \begin{tabular}{|l|}
-1 \\
\end{tabular} & \begin{tabular}{|l|}
-1 \\
\end{tabular} & -1 & -1 & -1 & 0 & 0 & 0 & 0 & 1 & 0 & 0 & 0 & \begin{tabular}{|l|}
-1 \\
\end{tabular} & 0 \\
\hline Schwefel 1.2 & 0 & 1 & -1 & -1 & -1 & -1 & -1 & -1 & -1 & -1 & 0 & -1 & 1 & 1 & 0 & 1 & \begin{tabular}{|l|}
-1 \\
\end{tabular} & -1 \\
\hline $\begin{array}{l}\text { Schwefel } \\
2.22\end{array}$ & 0 & 1 & 1 & \begin{tabular}{|l|}
-1 \\
\end{tabular} & \begin{tabular}{|l|}
-1 \\
\end{tabular} & -1 & 0 & 1 & -1 & 0 & 1 & 1 & 1 & 1 & 1 & 1 & 1 & 0 \\
\hline \begin{tabular}{|l} 
Schwefel \\
2.26 \\
\end{tabular} & 0 & 1 & 0 & \begin{tabular}{|l|}
-1 \\
\end{tabular} & \begin{tabular}{|l|}
-1 \\
\end{tabular} & -1 & -1 & -1 & -1 & -1 & -1 & -1 & 1 & 1 & 1 & 1 & 0 & -1 \\
\hline Sixhump & 0 & 0 & 1 & \begin{tabular}{|l|}
-1 \\
\end{tabular} & \begin{tabular}{|l|}
-1 \\
\end{tabular} & -1 & 0 & 0 & 0 & 0 & 0 & 1 & 0 & 1 & 1 & 1 & 1 & 0 \\
\hline Spherical & 0 & 0 & 1 & -1 & -1 & -1 & 0 & 0 & -1 & 0 & 0 & 1 & 1 & 1 & 1 & 0 & 1 & 0 \\
\hline Step & 0 & 1 & 1 & 0 & $\mid-1$ & -1 & 0 & 0 & -1 & -1 & 0 & 0 & 1 & 1 & 1 & 1 & 1 & 0 \\
\hline Zakharov & 0 & \begin{tabular}{|l|}
-1 \\
\end{tabular} & -1 & \begin{tabular}{|l|l|}
-1 \\
\end{tabular} & \begin{tabular}{|l|}
-1 \\
\end{tabular} & \begin{tabular}{|l|}
-1 \\
\end{tabular} & -1 & -1 & -1 & 0 & $\mid-1$ & -1 & 1 & 0 & \begin{tabular}{|l|}
-1 \\
\end{tabular} & -1 & \begin{tabular}{|l|l|}
-1 \\
\end{tabular} & -1 \\
\hline Total -1's & 2 & 1 & 8 & \begin{tabular}{|l|}
18 \\
\end{tabular} & 19 & 20 & 13 & 15 & 15 & 8 & 2 & 8 & 0 & 0 & 3 & 1 & 8 & 10 \\
\hline Total 0's & 18 & 6 & 3 & 2 & 1 & 0 & 7 & 4 & 2 & 9 & 11 & 5 & 4 & 3 & 5 & 4 & 3 & 9 \\
\hline Total 1's & 0 & 13 & 9 & 0 & 0 & 0 & 0 & 1 & 3 & 3 & 7 & 7 & 16 & 17 & \begin{tabular}{|l}
12 \\
\end{tabular} & 15 & \begin{tabular}{l|l|}
9 \\
\end{tabular} & 1 \\
\hline
\end{tabular}

GCPSO. This indicates that the rate at which the aBBPSO decreases its diversity can vary widely, possibly depending on the fitness landscape.

\section{Conclusions}

This paper proposed a measure to quantify the rate at which swarms, for different particle swarm optimisation (PSO) algorithms, decrease their diversity. The diversity rate-of-change (DRoC) measure is obtained by fitting two-piecewise 
linear approximations to diversity measurements taken at regular time steps. The proposed DRoC measure is the slope of the left of those two lines.

The DRoC measure was computed for different PSO algorithms for which there are intuitive expectations about the differences in behaviour in terms of decrease in diversity between the algorithms. The DRoC measure was shown to reflect those expected differences. Firstly, where one algorithm was expected to decrease its diversity faster than a second algorithm, the DRoC measure for the first algorithm was usually a statistically significantly lower negative value than for the second algorithm. Secondly, where no significant difference was expected between the rate at which two algorithms decreased their diversity, no statistically significant difference was usually found between the DRoC measures for the algorithms.

For each comparison, the results for some benchmark functions contradicted expectations. Furthermore, when comparing the alternative barebones PSO to the gbest PSO and the gbest GCPSO, the results varied widely for different benchmark functions. This could indicate that the fitness landscape has an influence on how algorithms decrease their diversity.

Future work will investigate the possible influence that the fitness landscape may have on the behaviour of swarms in terms of decreasing diversity.

Alternative measures can possibly be obtained form the two-piecewise linear approximations that were used to obtain the proposed DRoC measure. For example, using the angle between the slopes of the first and the second lines of the approximation might provide valuable information. Future work will investigate such alternative measures.

The DRoC measure for a simulation must be calculated after the simulation has completed. Methods will be investigated that allow the measure to be calculated in real time. Such methods can be used in algorithms where the diversity of a population is managed in real time, such as attractive-repuslive PSO [14].

\section{References}

1. Van den Bergh, F., Engelbrecht, A.P.: A new locally convergent particle swarm optimizer. In: Proceedings of the IEEE International Conference on Systems, Man, and Cybernetics. pp. 96-101 (2002)

2. Van den Bergh, F., Engelbrecht, A.P.: A study of particle swarm optimization particle trajectories. Information Sciences 176(8), 937-971 (2006)

3. Chen, M.R., Li, X., Zhang, X., Lu, Y.Z.: A novel particle swarm optimizer hybridized with extremal optimization. Applied Soft Computing 10(2), 367-373 (2010)

4. De Jong, K.A.: Analysis of the behavior of a class of genetic adaptive systems. Ph.D. thesis, University of Michigan, Ann Arbor, MI, USA (1975)

5. Eberhart, R.C., Kennedy, J.: A new optimizer using particle swarm theory. In: Proceedings of the Sixth International Symposium on Micro Machine and Human Science. vol. 1, pp. 39-43. New York, NY (1995)

6. Engelbrecht, A.P.: Computational intelligence: an introduction. John Wiley \& Sons (2007) 
7. Engelbrecht, A.P.: Scalability of a heterogeneous particle swarm optimizer. In: Proceedings of the 2011 IEEE Symposium on Swarm Intelligence. pp. 1-8. IEEE (2011)

8. Fan, S.K.S., Chang, J.M.: Dynamic multi-swarm particle swarm optimizer using parallel PC cluster systems for global optimization of large-scale multimodal functions. Engineering Optimization 42(5), 431-451 (2010)

9. Kennedy, J.: Bare bones particle swarms. In: Proceedings of the 2003 IEEE Swarm Intelligence Symposium. pp. 80-87. IEEE (2003)

10. Kennedy, J., Eberhart, R., et al.: Particle swarm optimization. In: Proceedings of IEEE International Conference on Neural Networks. vol. 4, pp. 1942-1948. Perth, Australia (1995)

11. Kennedy, J., Mendes, R.: Population structure and particle swarm performance. In: Proceedings of the 2002 IEEE World Congress on Computational Intelligence. vol. 2, pp. 1671-1676. IEEE Computer Society (2002)

12. Kennedy, J.F., Kennedy, J., Eberhart, R.C.: Swarm intelligence. Morgan Kaufmann (2001)

13. Mishra, S.: Some new test functions for global optimization and performance of repulsive particle swarm method. Tech. rep., University Library of Munich, Germany (2006)

14. Monson, C.K., Seppi, K.D.: Adaptive diversity in PSO. In: Proceedings of the 8th annual conference on Genetic and evolutionary computation. pp. 59-66. ACM (2006)

15. Olorunda, O., Engelbrecht, A.P.: Measuring exploration/exploitation in particle swarms using swarm diversity. In: Proceedings of the 2008 IEEE Congress on Evolutionary Computation (IEEE World Congress on Computational Intelligence). pp. 1128-1134. IEEE (2008)

16. Peer, E.S., Van den Bergh, F., Engelbrecht, A.P.: Using neighbourhoods with the guaranteed convergence PSO. In: Proceedings of the 2003 IEEE Swarm Intelligence Symposium. pp. 235-242. IEEE (2003)

17. Price, K., Storn, R.M., Lampinen, J.A.: Differential evolution: a practical approach to global optimization, chap. Appendix A.1: Unconstrained uni-modal test functions, pp. 514-533. Natural computing series, Springer, Berlin, Germany (2006)

18. Rahnamayan, S., Tizhoosh, H.R., Salama, M.: A novel population initialization method for accelerating evolutionary algorithms. Computers \& Mathematics with Applications 53(10), 1605-1614 (2007)

19. Suganthan, P.N.: Particle swarm optimiser with neighbourhood operator. In: Proceedings of the 1999 Congress on Evolutionary Computation. vol. 3. IEEE (1999)

20. Tang, K., Yao, X., Suganthan, P.N., MacNish, C., Chen, Y.P., Chen, C.M., Yang, Z.: Benchmark functions for the CEC 2008 special session and competition on large scale global optimization. Tech. rep. (2007)

21. Yao, X., Liu, Y., Lin, G.: Evolutionary programming made faster. IEEE Transactions on Evolutionary Computation 3(2), 82-102 (1999) 